\title{
Challenges in Characterizations of MTJ Thin Film Stack for Spin-transfer Torque MRAM Device by Analytical TEM in Wafer-foundries
}

\author{
Wayne Zhao, Hemant Dixit, Travis Mitchell and William Taylor \\ GLOBALFOUNDRIES US Inc., Malta, New York, United States
}

Magnetoresistive Random Access Memory (MRAM) is a non-volatile memory device. The unique cell structure of a MRAM device opens an era of a smaller memory chip size at a given technology node, compared to other RAM devices, by enabling higher densities at a lower cost [1 3]. Among several MRAM memory cells, a spin-transfer torque MRAM (STT-MRAM) has the potential to become a leading storage technology as a high-performance memory. In terms of scaling at the 1x nm nodes, STT-MRAM potentially challenges dynamic-RAM (DRAM) and static-RAM (SRAM); and for IoT and mobile applications, MRAM is replacing embedded NOR flash memory. In a STT-MRAM device, to flip the spin of the electrons in one of the ferromagnetic layer by transferring the angular momentum, a spin-polarized current is applied through a vertical stack of ultrathin films, which consists of two ferromagnets separated by oxide layer(s), known as Magnetic Tunnel Junction (MTJ). Subsequently, a smaller applied current can be used to sense either low or high resistance depending on the parallel or antiparallel orientation of the two ferromagnetic layers, and this precisely serves as memory in terms of either low resistance state ("ON") or high resistance state ("OFF") [1-3]. In particular, a perpendicular MTJ (pMTJ) with high reliability at high temperatures, in which the magnetic moments are perpendicular to the plane of the layers is more scalable compared to an in-plane MTJ (iMTJ). Therefore, pMTJ STT-MRAM is a more promising technology to replace DRAM and other memory technologies than other types of memories. The core of a MTJ stack is composed of $\mathrm{MgO}$ and ferromagnetic $(\mathrm{CoFeB})$ layers, with various thickness of few atomic layers, stacking sequence, and boron concentrations, pertinent to the needs of structural integrity, yield enhancement, and device performance, for each particular technology node during a high-volume manufacturing (HVM). Engineering to tailor desired geometry, layer thickness and atomic-alignment from film to film to ensure the effectiveness of free layer (FL) versus the stability of the fixed layer are vital to the performance of these pMTJ device. Thus, to ensure the device performance, analyses from physical failure analysis (PFA) are critical to identify process induced variations within MTJ stack as feedbacks to tweak process conditions for device performance optimization. Here, we discuss some of the challenges and solutions for effective characterizations using analytical transmission electron microscopy (Analytical TEM).

Figure-1a is an illustration of a MRAM device [3]. Analytical TEM examples are demonstrated in Figs$1 \mathrm{~b} \sim 1 \mathrm{j}$. Major challenges include the following. First is the difficulty to precisely align crystal zone in these ultrathin films and define film boundaries. Since the MRAM is typically embedded in the back-end-ofline (BEOL) of the nanometer device, Fig-1b. TEM foil buckling at far BEOL against the reference from crystal zone well aligned based on Si wafer substrate, is usually an annoyance but still within manageable tolerance range, for BEOL trenches with dozens of nanometers. However, this cannot be overlooked when to characterize the MTJ films with only few monolayers of atoms, since the buckling can completely ruin the effectiveness of ultrathin MRAM films in the TEM sample. $\mathrm{MgO}$ lattice is a $\mathrm{NaCl}$ type at ambient temperatures, and its lattice matching with $\mathrm{CoFeB}$ is critical for understanding the MTJ performance. However, since a) there are few major crystal zones for $\mathrm{NaCl}$-typed $\mathrm{MgO}$, and b) vertical dimension is only a few atomic-layers, this is challenging for nano-beam diffraction. Second, associated with aforementioned, is the ambiguity in interpretation of intermixing extent across the film boundaries, versus artifacts associated with the delocalization of the x-ray energy dispersive spectroscopy (XEDS). In theory, to minimize electron channeling effect during XEDS, it is better to tilt the TEM foil about 2 degree off a 
crystal zone, per learning from Si lattice. However, for the ultrathin MTJ films, the consequence of slightly off crystal zones is the broadening of the XEDS profiles for elements of interests. Third, there is a dilemma between the need for almost atomic-resolution XEDS mapping on these MTJ films, with few monolayers of atoms, against the unavoidable XEDS beam broadening induced delocalization. This problem deters extensive attempts to apply STEM-XEDS electron tomography. Finally, we have the problem of geometries (cylindrical shapes) in a real patterned MRAM device. These make interpretation of XEDS intensities difficult - is the signal pure chemical contributions or is it geometry contributions, e.g., purely MTJ at the center of the TEM foil, while edge somewhat a mixture of MTJ and dielectric, Fig.-1e. Resultantly, the weight of these challenging factors impact more in characterizations of ultrathin MTJ film stack, than large features of BEOL trenches or Front-End-Of-Line (FEOL) stressor such as SiGe/SiP, with relative impact scale illustrated in Fig-1k [4].

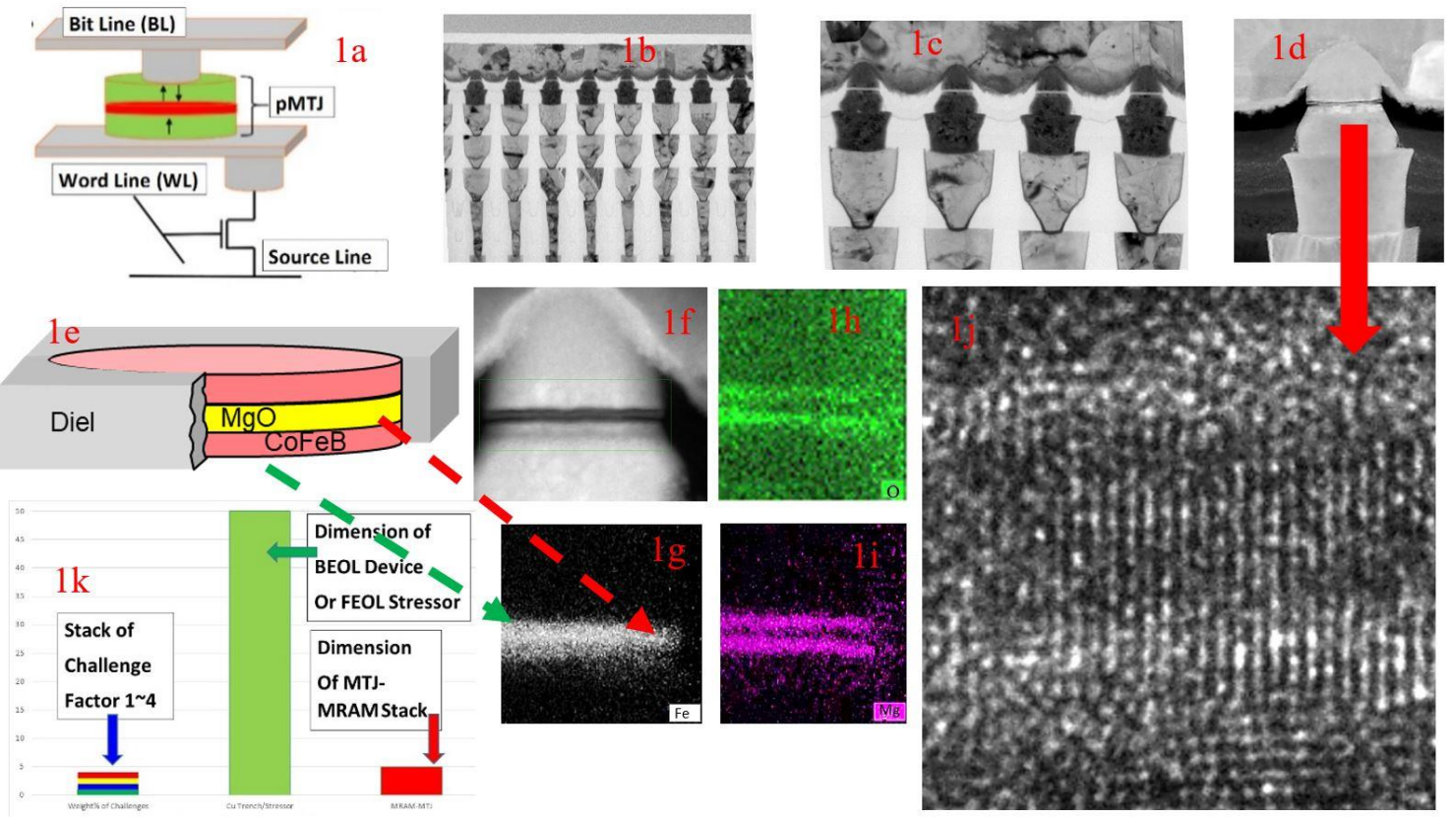

Figure 1. (a) Schematic of MTJ in MRAM [3]; (b) (d) cross-sectional TEM and STEM of MRAM device; (e) geometry of TEM foil vs. MRAM device; (f) (i), XEDS mappings for the MTJ stack; (j) HRTEM on MTJ films; and (k) illustration challenging factors against device dimensions.

\section{References}

1. D. Apalkov, et al., Proceedings of the IEEE, 104-10, (2016), pp. 1796-1830.

2. B. Naik, et. al. International Electron Devices Meeting (IEDM), (2019), in-print.

3. Hemant Dixit, et al., IEEE International Magnetic Conference (INTERMAG), (2018), pp. 1-1.

4. Thanks to Frieder Baumann at Fab8 for inspiring discussions, and Management and Legal teams for supporting the publication clearance. 